\title{
LOS RETOS DE LAS NUEVAS TECNOLOGÍAS PARA LA EDUCACIÓN A DISTANCIA
}

\section{The challenges of the new technologies for the distance}

\author{
Jaume SARRAMONA i LÓPEZ \\ Universitat Autònoma de Barcelona. Departamento de Pedagogía Sistemática y \\ Social. G-6.08193 Bellaterra. Barcelona. Correo-e: j.sarramona@uab.es
}

Fecha de aceptación definitiva: marzo 2001

BIBLID [(1130-3743) 12, 2000, 13-27]

RESUMEN

La tecnología ha penetrado en todos los ámbitos de la vida actual y, con mayor motivo, en la educación a distancia. La aplicación de las nuevas tecnologías ha modificado el concepto mismo de "distancia", al facilitar la comunicación inmediata y la interacción. Pero la correcta aplicación de las nuevas tecnologías en la educación a distancia precisa de condiciones ineludibles: existencia de un modelo didáctico coherente, preparación previa en los destinatarios, planificación a cargo de equipos multidisciplinares, establecimiento de las condiciones para la interacción social y con el mismo material didáctico, fomento de la motivación, etc. En cualquier caso, la libertad de iniciativa en el sujeto que aprende no exime de garantizar la adquisición de los conocimientos y competencias fundamentales en el campo objeto de estudio.

Descriptores: nuevas tecnologías, educación a distancia, interacción, planificación, motivación.

\section{SUMMARY}

The technology has penetrated in all the environments of the current life and, with more reason, in the education at distance. The application of the new technologies has 
modified the same concept of "distance", when facilitating the immediate communication and the interaction. But the correct application of the new technologies in the education at precise distance of unavoidable conditions: existence of a coherent didactic model, previous preparation in the addressees, planning in charge of multiprofessionals teams, establishment of the conditions for the social interaction and with the same didactic material, I foment of the motivation, etc. Anyway, the initiative freedom in the fellow that learns doesn't exempt of guaranteeing the acquisition of the knowledge and fundamental competitions in the field study object.

Key words: new technologies, education at distance, interaction, planning, motivation.

\section{LA TECNOLOGÍA COMO CARACTERÍSTICA SUSTANCIAL DE LOS TIEMPOS ACTUALES ${ }^{1}$}

Nos resultaría difícil siquiera imaginar cómo sería la vida, el trabajo y el conjunto de la sociedad sin los recursos que la tecnología ha puesto a nuestra disposición. Por ello no tiene ningún sentido abrir debates sobre la bondad o no de la tecnología en general, puesto que su presencia resulta tan necesaria como inevitable. La cuestión estriba en decidir qué uso vamos a darle en cada ámbito de nuestra vida y cómo nos conviene utilizarla.

Como decimos, sería difícil encontrar ámbitos donde la tecnología no estuviera presente de alguna forma, si bien no ha logrado en todos igual nivel de penetración y, por supuesto, existen notables diferencias entre territorios, grupos sociales y personas. Observemos que algo tan íntimo como la comunicación interpersonal se ha visto sometida a cambios radicales con la incorporación intensiva del teléfono -fijo o móvil- y la informática. No hará falta insistir nuevamente en la justificación de calificar la sociedad actual, y aún más la futura, como la sociedad de la comunicación y de la información. Pero, ¿qué ocurre con la educación?

Hay que reconocer que en general la actividad educativa no ha incorporado el nivel de tecnología que potencialmente cabría esperar, aun siendo una forma de comunicación interpersonal de carácter fundamental para los individuos y las sociedades. Las razones son diversas, desde las estrictamente económicas hasta las corporativas y de mentalización por parte del profesorado. Tampoco se trata de solicitar la tecnificación de la educación de manera absoluta, sin análisis reflexivo sobre sus posibilidades y limitaciones, sino de advertir en qué medida la tecnología puede colaborar en la consecución de las metas que la educación tiene como propias.

En esta ocasión, el análisis reflexivo sobre el papel que puede y debe tener la tecnología en la vertiente que compete a la comunicación y la información lo limitaremos

1. Este texto corresponde a la conferencia pronunciada en el IX Encuentro sobre Educación a Distancia que organizó la Universidad de Guadalajara (México) en noviembre del 2000. 
a la modalidad de la "educación a distancia", si bien muchas de las conclusiones que pudieran obtenerse resultan igualmente válidas para la educación presencial, que hoy advierte cómo el ímpetu de los avances tecnológicos la impulsan hacia cambios profundos, tal como señalan multitud de autores ${ }^{2}$.

\section{¿QUÉ SUPONE PARA LA EDUCACIÓN A DISTANCIA LAS NUEVAS TECNOLOGÍAS?}

La primera consecuencia para la educación a distancia de la aplicación de las denominadas "nuevas tecnologías", representadas básicamente por el ordenador multimedia y la telecomunicación informática, es la revisión misma del concepto de "distancia", que queda más limitado al estricto campo espacial. Gracias a ellas el proceso de enseñanza puede llegar a ser sincrónico con el de aprendizaje, sincronía que se puede extender al feed-back y a la tutoría, tradicionalmente diferidos en una modalidad didáctica que emplee los medios de comunicación más convencionales. Así se justifica que se abra camino la denominación de "autoformación" para hacer referencia a las situaciones estructuradas de aprendizaje sin la presencia física inmediata del docente (Sarramona, 1999), si bien la más tradicional de "educación" o "enseñanza" a distancia seguirá teniendo vigencia en el vocabulario pedagógico 3 .

La posibilidad de interacción y de respuesta inmediata a las consultas de los alumnos constituyen la primera gran aportación de las nuevas tecnologías a la educación no presencial. Pero la potencialidad pedagógica no queda reducida a estos apartados. Todo parece indicar que los cambios promovidos por la tecnología multimedia $^{4}$ que proporciona la informática puede llevar a cambios profundos en la manera de aprender, puesto que se trata de los mismos recursos que se hallan en los lugares de trabajo y en los hogares para llevar a cabo la actividad laboral, el acceso a la información y el ocio.

Es creciente la sustitución en muchos programas de los "viejos" materiales impresos por los nuevos materiales informatizados en las correspondientes Web, pero aún son abundantes las instituciones de educación a distancia que siguen teniendo en los materiales impresos su principal medio de soporte didáctico. Por otra parte, no siempre resulta evidente la mejora que las nuevas

2. Steve WHEELER (2000), por ejemplo, refiriéndose a tales cambios aplicados al nivel universitario señalaba en el título de una ponencia: "The traditional university is dead: long live the distributed university".

3. También se abren camino nuevas perspectivas metodológicas con el denominado distributed learning, que incluye aprendizaje a distancia, métodos didácticos flexibles y abiertos provocados por los nuevos recursos del aprendizaje, al tiempo que se incorporan los mejores esquemas del aprendizaje tradicional.

4. Algunos proponen la denominación de "hiper-media" cuando se trata de una combinación de medios que no toma un formato no lineal, en sustitución de la ya tradicional de "multi-media", pero aquí se seguirá utilizando esta última por ser más común, con la advertencia que la diversidad de medios presentados a través del ordenador efectivamente adopta la estructura de combinación no lineal. 
tecnologías aplicadas a ciertos programas aportan respecto al uso de los materiales convencionales. $Y$ es que las condiciones necesarias para materializar una propuesta didáctica que explote al máximo las posibilidades de las nuevas tecnologías no son tan fáciles de alcanzar. Veamos algunas de las principales.

\section{1) Necesidad de un modelo didáctico coherente}

Aunque pueda resultar una obviedad, es preciso recordar de entrada que el soporte de los materiales didácticos, si bien introduce indudables factores de condicionalidad - recuérdese una vez más a MacLuhan con lo de "el medio es el mensaje»- por sí mismos no son lo fundamental para determinar la calidad y eficacia de las informaciones ofrecidas, sino el modelo de enseñanza-aprendizaje que ha guiado su diseño y contenido.

Tiempo atrás, fue en la educación a distancia donde se llevó a cabo la materialización más completa de los principios defendidos por la "tecnología educativa" de base conductista. El caso de los materiales inicialmente confeccionados por la Universidad Abierta de Venezuela puede constituir un ejemplo significativo de ello. Y es que la elaboración de unos materiales que debían contener todos los componentes del acto didáctico, sin estar sometidos a la dinámica y diversidad de respuestas que producen en la docencia presencial, facilitó la proyección completa de un modelo que se presentaba como exhaustivo en este campo: objetivos predefinidos, contenidos vinculados, actividades, evaluación... etc. El modelo conductista, por otra parte, proporcionaba seguridad a los autores de los materiales sobre el camino a seguir en su confección. El tipo de aprendizaje que se proponía era de carácter lineal, congruente con las posibilidades intrínsecas del medio impreso, pero también de los complementos audiovisuales al uso. La generalmente demandada autosuficiencia de los materiales para lograr las metas propuestas favorecía esta estructura.

La superación de las teorías puramente conductistas y el desarrollo de concepciones del aprendizaje donde el sujeto tiene un papel más activo y se erige en reelaborador del conocimiento puso en crisis el modelo anterior, abriéndose camino las propuestas didácticas mucho más dinámicas y también más complejas de materializar.

Principios del cognitivismo, en sus diversas formas, y de lo que se ha difundido con la denominación genérica de "constructivismo" se han acreditado como imprescindibles para lograr un aprendizaje significativo, firme y creativo. Así surgen en los diseños didácticos elementos como: organizadores de avance, mapas conceptuales, redes simbólicas, procesos de inducción, contra-ejemplos provocadores de disonancias cognitivas, actividades creativas, etc. Todo ello, hay que decirlo, sin olvidar algunos de los elementos clásicos de las teorías conductistas 
como la fijación de objetivos previos (aunque no exclusivamente operativos), reforzadores y feed-backs proporcionados por las pruebas de autoevaluación ${ }^{5}$.

Sin embargo, en la práctica resulta difícil encontrar materiales didácticos que ofrezcan un modelo estructurado de todos los elementos indicados. Los autores tienen dificultades en su confección y los organismos responsables de los programas a distancia habitualmente no les ofrecen la ayuda y formación necesaria en este terreno; todo lo más se limitan a proporcionarles la lista de elementos y ejemplos ilustrativos de los mismos.

Sin duda lo que hoy sabemos sobre el aprendizaje nos exige aplicar modelos didácticos que sean coherentes con los principios reseñados, pero ocurre que tales modelos, al contrario de lo que ocurría con los técnico-conductistas, son más coherentes con una situación de interacción permanente, como la que puede ofrecer la enseñanza presencial, que la proporcionada por unos materiales preelaborados. Y con ello no se está diciendo que en la enseñanza presencial se prodigue ampliamente la forma de actuación docente que demandan las propuestas cognitivo-constructivistas, pero existe mayor reflexión y desarrollo al respecto.

El desafío al que se enfrenta la educación a distancia en general y la realizada a través de las nuevas tecnologías de la información y la comunicación (NTIC) es precisamente el desarrollar modelos instructivos (si se quiere mantener el término) que sean coherentes con las teorías vigentes del aprendizaje y factibles de ser llevados a la práctica por parte de los responsables de confeccionar los programas.

\section{2) Exigencias multidisciplinares en la confección de los materiales didácticos}

En la educación a distancia siempre se ha demandado la necesidad de constituir equipos multidisciplinarios para elaborar los materiales didácticos, aun los impresos. Junto al denominado "experto en contenidos" se han solicitado un "diseñador instructivo" y un "experto en medios" para la inclusión de los recursos audiovisuales.

Con el empleo de las nuevas tecnologías resulta imprescindible la presencia del experto informático junto al autor convencional y el didacta especializado en la enseñanza a distancia. También aparece la figura del diseñador gráfico, que actúa sobre los elementos formales de los materiales tecnológicos, al igual que ya actuaba en el momento de la edición de los materiales escritos, si se quería una

5. Como es bien sabido, la concepción constructivista del aprendizaje recoge una amalgama de teorías diversas, entre las cuales están las desarrolladas por Vygotsky durante el primer tercio del siglo $\mathrm{xx}$ y que, sin duda, tienen en gran parte validez actual, aunque algunos de sus planteamientos se oponían claramente a los defendidos por Piaget (VyGotsky, 1973) el otro gran autor recuperado. Sin embargo, cuando se habla de constructivismo en el aprendizaje mediante las NTIC a veces se fuerza la interpretación de estos autores que, lógicamente, no desarrollaron sus teorías en el marco tecnológico actual. 
calidad gráfica destacable. En este caso, el diseñador gráfico actuará sobre la Web de acceso a la información inicial y sobre la presentación del conjunto de los elementos que constituyen el programa.

La novedad y complejidad del medio informático a los ojos de la gran mayoría de los docentes lleva en cierto modo a su mitificación. Los textos impresos y los medios audiovisuales, aunque con ciertas dificultades estos últimos, han sido recursos empleados en la docencia convencional en los centros escolares presenciales de todos los niveles, de modo que no resultan extraños a los docentes. Pero ante el material informático se hallan en situación de desventaja y forzosamente han de recurrir a las directrices impuestas por los profesionales de ese campo, con la inevitable dependencia - y costo añadido- que ello comporta.

Ante la elaboración de materiales didácticos informatizados, el técnico en informática toma un papel protagonista, hasta el punto de condicionar en gran manera todo el proceso. Sin excluir su presencia, lógicamente, se hace necesario demandar a los docentes responsables de elaborar los materiales didácticos conocimientos suficientes sobre las posibilidades, limitaciones y costos del medio para realizar propuestas optimizadoras y factibles.

Pero cabe ir más allá. La difusión generalizada de las nuevas tecnologías en la educación y formación, y más la adquisición de esta última bajo el principio de justin-time (Romiszowski, 1999), hará que sólo una pequeña parte de las informaciones accesibles hayan sido confeccionadas por equipos multidisciplinarios de técnicos, quedando el experto en diseño instruccional como el más prescindible. La consecuencia será una pléyade de informaciones con diversos niveles de estructuración y contenido que deberán ser seleccionadas por el propio usuario. En algunos casos se podrá decir que esa selección personal es conscientemente pretendida desde el programa, pero en otros muchos será el resultado de la falta de planificación coherente.

\section{3) Habilidades específicas en los destinatarios}

La generalización de las nuevas tecnologías puede comportar también el caos informativo, ante el cual será imprescindible la posesión de habilidades personales para buscar las informaciones relevantes, las vinculadas a las propias necesidades, para darles coherencia informativa, etc., todo lo cual demanda competencias básicas bien afincadas, conocimiento de las fuentes informativas, disciplina personal y, sobre todo, capacidad de síntesis creativa.

Así reaparece nuevamente la necesidad de preparar a los usuarios de la educación a distancia para la utilización de las nuevas tecnologías, del mismo modo como se les ha preparado, a través de programas previos en "técnicas de estudio a distancia", para el estudio mediante los medios convencionales, en especial el 
medio impreso ${ }^{6}$. La naturaleza de la preparación para el estudio mediante las nuevas tecnologías deberá ser sensiblemente diferente, puesto que deberán tratar las técnicas de búsqueda de información a través de la Web, venciendo, por ejemplo, la tendencia habitual al zapping que se ha desarrollado ante la televisión multicanal. Si se emplea el hipertexto de manera generalizada el destinatario deberá ser un lector activo, que selecciona pero que también establece itinerarios personales en el camino del conocimiento (Landow, 1997), etc.

Ante las inmensas posibilidades que ofrecen las nuevas tecnologías, con redes abiertas y cerradas que muestran múltiples y diversas informaciones, sólo las personas bien preparadas para su uso conseguirán obtener beneficio significativo para su formación personal. Otra cosa es el campo del ocio y de la información puntual o esporádica, donde no resulta necesario el logro de aprendizajes firmemente estructurados.

Al lado de las habilidades comentadas no se puede dejar de lado el factor económico. Cierto que los costos de los materiales informáticos resultan accesibles para una gran parte de la población de los países medianamente desarrollados, pero en comparación con los costos de los materiales tradicionales resultan selectivos para acceder a la educación a distancia, lo cual contrasta con una de las metas tradicionales de esta modalidad educativa, que ha sido la de llegar a las poblaciones con menos recursos. No insistiremos tampoco, porque es sobradamente conocido, que los recursos informáticos quedan obsoletos en breve tiempo y cada vez se piden aparatos más potentes y rápidos para atender al creciente aumento de las exigencias del aprendizaje a través de la red.

En justa contrapartida, es igualmente cierto que los materiales didácticos de la Web ofrecen la ventaja de poder atender a un número infinito de usuarios y poder ser modificados de forma rápida, en contraposición a los materiales editados, sea cual sea el soporte empleado, desde el papel al cD.

\section{4) La comunicación interpersonal}

Son bien conocidas las posibilidades que ofrecen las nuevas tecnologías informáticas para la comunicación interpersonal, sea sólo por escritura, en el correo electrónico, sea también mediante imágenes, en la videoconferencia o equivalente.

Este hecho ha supuesto una auténtica revolución en el tradicional contexto de soledad que ha caracterizado la educación a distancia. Aunque ya existe desde hace tiempo la vídeoconferencia a través de la tecnología de la televisión, la incorporación de la informática la ha generalizado hasta el punto de llevarla hasta el mismo domicilio del usuario.

6. Es fácil señalar que de las técnicas clásicas de subrayar en el texto, por ejemplo, se deba insistir en la identificación de los enlaces más relevantes de la información ofrecida para no perderse en un mar informativo. 
Los beneficios más valorados de la enseñanza tradicional en aula son potencialmente incorporados a la enseñanza a distancia, puesto que se hace factible el debate en pequeño grupo, la consulta instantánea al profesor, la ayuda al compañero e incluso la realización en grupo de ciertas tareas on line. Todo ello lleva a insistir que el nivel de personalización de las interacciones es superior al habitual de las aulas, al tiempo que las relaciones sociales pueden llegar a todos los rincones del planeta, con la sola condición de compartir idioma.

Admitido todo lo anterior, tampoco se pueden olvidar ciertos condicionantes propios de las situaciones específicas de la comunicación informática. En primer lugar, y a riesgo de recordar una obviedad, la comunicación telemática nunca es equivalente a la comunicación "cara a cara"; el aula virtual, con todas sus indiscutibles posibilidades, no es una aula real. Y ello se subraya no tanto para hacer el paralelismo entre la enseñanza a distancia que nos ocupa y la enseñanza presencial, cuanto para advertir que las reuniones periódicas presenciales que generalmente se programan en la metodología a distancia para lograr objetivos de socialización y motivación siguen siendo necesarias y no quedan plenamente sustituidas por la comunicación telemática.

Pero hay otra perspectiva del tema. Frente a la soledad que puede ser vivida angustiosamente por algunos participantes en los programas a distancia, hasta el punto de que lleguen a abandonar por esta causa, existen personas que eligen la modalidad a distancia precisamente porque prefieren el estudio en solitario, con todo lo que ello supone de libertad en el ritmo de aprendizaje, en cuanto al lugar y horario, etc. Tales personas, que pueden tener cubiertas las dimensiones de formación social por otros conductos, se pueden sentir incómodas ante la obligación de conectarse frecuentemente al correspondiente chat. Una cosa es favorecer y posibilitar la interacción y otra forzar a los sujetos a realizar comparecencias periódicas que no sean imprescindibles para el aprendizaje pretendido. En esta situación el exceso de interactividad puede llegar a ser contraproducente, de modo que será recomendable combinar situaciones obligatorias de interacción grupal con otras plenamente voluntarias y no confundir la posibilidad de interacción con su obligatoriedad intensiva en cualquier circunstancia.

Adviértase también que las comunicaciones instantáneas no son siempre necesarias para lograr la deseada interacción personal, siempre y cuando el tiempo transcurrido entre los mensajes sea breve. Los participantes en un programa de educación a distancia comprenden perfectamente que una cosa es el tiempo preciso para mandar un mensaje a su profesor, tutor o compañeros de estudio y otra distinta el tiempo hábil de que dispongan aquéllos para las respuestas. 


\section{5) El aprendizaje como proceso interactivo}

La interacción comentada hacía referencia a la comunicación entre personas pero es evidente que el aprendizaje mismo puede ser contemplado como un proceso de interacción cognitiva, materializándose así uno de los principios básicos del constructivismo, tomado principalmente de la teoría piagetiana. Y éste puede ser uno de los puntos fuertes de las posibilidades pedagógicas de las nuevas tecnologías. El aprendizaje no se consigue con la simple acumulación de conocimientos, sino mediante un proceso dinámico que da lugar a redes conceptuales y no a listados de informaciones

Sin embargo, la posible interacción requiere de la correspondiente estructuración de las informaciones ofertadas. La simple presencia de un texto convencional colocado en la Web, cuya lectura se efectúa tras el visionado de pantallas sucesivas, no proporciona más interacción que la ofrecida por el mismo texto impreso sobre papel; tanto es así que es muy probable que en tales circunstancias el interesado opte por imprimirlo y proceder a su lectura en el papel.

Tampoco se puede hablar de interacción plena cuando las nuevas tecnologías se emplean estrictamente para ampliar lo que constituye una situación típica de aula, esto es, unas sesiones expositivas por parte del docente que pueden llegar hasta el domicilio de los alumnos a través de la teleconferencia. Aunque se posibilite la intervención de éstos y el interrogatorio directo a cargo del docente, el proceso no supera el de los modelos clásicos de interacción limitada.

Un primer nivel de interacción en el material es el ofrecido por el hipertexto, en cuanto posibilita la ampliación de informaciones, ofrece información complementaria, aclara conceptos, etc. El hipertexto, en tanto que entramado de núcleos de información (nodos), permite una secuencia en razón de las preferencias del usuario. Es el tipo de información que se adopta en Internet y en las enciclopedias informatizadas, por ejemplo. Ahora bien, tampoco se trata de abusar del diseño en el sentido de multiplicar los nodos excesivamente, produciendo sobreabundancia de enlaces, ni dejarlos sin conexión lógica entre sí, porque con ello se crea desorientación, ansiedad e incertidumbre (Rodríguez Neira, 2000). Pero la interacción plena exige ir más allá, con lo cual llegamos a los condicionantes que lo hagan posible: por una parte unos medios tecnológicos potentes y por otra un material pensado para tal fin.

Si se pretende un aprendizaje realmente estructurado, el material didáctico presente en la Web no puede estar situado de manera simplemente acumulativa. Por una parte se necesita una organización de la información que puede tomar la forma de mapa conceptual, por ejemplo7, para garantizar la comprensión, y por otro ha de hacer posible la interacción para garantizar un aprendizaje activo.

7. Un grupo de profesores de la Universidad de Oviedo ha desarrollado un programa para procesar información mediante hipertexto basado en los mapas conceptuales y que denominan "hypertexto" (Állvarez y otros, 2000). 
El punto clave para lograr la interacción que lleve al aprendizaje realizado de forma integradora, "reconstruyendo" la información de acuerdo a las necesidades y estrategias personales, lo constituye el tipo de actividades que los alumnos se vean precisados a realizar. No se trata simplemente de "navegar" por la red, aunque es preciso dominar la técnica de cómo hacerlo, sino de tener que analizar, comparar, sintetizar, aplicar, etc.; en definitiva aquel conjunto de actividades que superan la simple interiorización y comprensión de las informaciones.

En la misma medida que la interacción supone personalización del proceso, se hace necesario incorporar la evaluación, especialmente en su perspectiva "auto", para obtener feed-backs inmediatos y orientadores de la eficacia en el aprendizaje. Y ello debiera estar inserto en el proceso mismo de aprendizaje, y no como un apéndice del cual incluso se puede prescindir. Indicar los errores, dirigir hacia informaciones complementarias, demandar nuevas actividades... son otras tantas opciones que proporcionan el feed-backs activo.

La interacción demandada ha de dar satisfacción a la realidad de diversas capacidades y formas de aprender por parte de los sujetos, para que efectivamente se materialice el principio del "aprendizaje situado" (Lave y Wenger, 1991). Por consiguiente, amén de la posibilidad de distintos niveles de profundización en el aprendizaje, se han de ofrecer distintos caminos para llegar a él. Esto supone mostrar los mismos contenidos de distintas formas, incluir ejemplos diversos, hacer referencia a múltiples contextos, permitir la elección entre posibles actividades, etc. En definitiva volvemos al condicionante ya indicado de un diseño didáctico que adopte estas posibilidades.

\section{6) Posibilidades para la simulación}

Las denominaciones de "aula virtual", "campus virtual", etc., se han hecho ya habituales con la difusión de las NTIC y no sólo entre los programas e instituciones de educación a distancia sino también en las presenciales. Sin duda ha sido el uso generalizado de esas tecnologías las que han contribuido decisivamente a romper la frontera entre la educación presencial y la no presencial, puesto que las redes telemáticas existen en ambos casos. Ninguna universidad presencial de mediano prestigio carece hoy de su propia intrarred.

El desarrollo de la realidad virtual, tan evidente en el caso de los videojuegos, ofrece indudables posibilidades para la formación a distancia a través de la simulación de diversas dimensiones propias de la preparación profesional. Y no se piense que se trata solamente de la preparación en rutinas, por otro lado cada vez menos relevantes en el mundo laboral avanzado, sino especialmente de la preparación para un ejercicio profesional reflexivo, donde lo más importante es el análisis simbólico y la toma de decisiones de manera innovadora. No se olvide que las empresas más avanzadas son las que tienen al conocimiento como objeto de tratamiento. 
Es en este sentido que los entornos virtuales ofrecen un espacio diversificado para la formación (Pagés, 1999). El ya tradicional estudio de casos, por ejemplo, cobra nuevas dimensiones al disponer de una información amplia y poder lograr una adaptabilidad máximamente individualizada. Bastaría recordar el diagnóstico por ordenador en medicina, la localización de averías en mecánica, los combates simulados en el terreno militar, etc., y el campo se extiende ahora al mundo de la economía, del derecho, de la agricultura, de la química...

La realidad virtual permite la simulación de las situaciones reales complejas sin los riesgos ni costos de la formación "in situ", por lo cual sus ventajas son indiscutibles, pero nuevamente es preciso recordar que la realidad virtual no es la realidad compleja en que vivimos. Sin necesidad de entrar ahora en serias disquisiciones filosóficas sobre el tema, porque indudablemente la virtualidad es un tipo de realidad, situados en el terreno pedagógico de preparar para la vida ordinaria, habrá que prever la transferencia de los aprendizajes logrados en realidades virtuales a las ordinarias, de modo que no se podrán eliminar las prácticas en estas últimas, lo que denominaríamos como "prácticas reales".

\section{7) ¿Y qué decir de la motivación?}

Siempre se ha indicado que la motivación constituye un requisito para el aprendizaje, si éste se concibe como una actuación libre y guiada por el propio sujeto que aprende. Sólo una actuación externa, decidida y constante, al estilo de la ejercida por los docentes en situación de aula, puede compensar parcialmente la falta de motivación inicial, pero aun en ese caso con resultados inciertos.

Tratándose del aprendizaje no presencial, donde la intervención directa del docente es más limitada — cuando existe- y dado que los alumnos son personas adultas, la motivación resulta decisiva para el éxito del sistema. Mantener la motivación inicial que se da por supuesta cuando el sujeto se incorpora voluntariamente a un programa ha sido siempre objeto de preocupación básica para la educación a distancia.

La primera fuente de mantenimiento de la motivación serán los contenidos objeto de aprendizaje. Contenidos que han de responder a los intereses y necesidades de los sujetos adultos, porque éstos no admitirán informaciones que no estén justificadas en razón de tales intereses o que no sean los idóneos para la resolución de problemas concretos. Por consiguiente, la adecuada selección de contenidos no sólo constituye una exigencia de eficacia sino también de motivación, con independencia de los medios empleados en el proceso de enseñanza-aprendizaje. Los contenidos serán adecuados cuando resuelvan los problemas de los sujetos que aprenden, esto es, sean pertinentes a los objetivos propuestos y hagan referencia a contextos próximos o conocidos, de modo que muestren que han sido determinados pensando en la realidad de los destinatarios. 
El modelo didáctico empleado incide directamente sobre la motivación, en especial en dos de sus elementos: la forma como fomenta el aprendizaje y la posibilidad de verificar los resultados obtenidos. Nuevamente el dinamismo en el proceso de aprendizaje aparece como requisito. Se trata de la interactividad ya comentada con la materia objeto de estudio y con los sujetos participantes en el sistema: docentes, tutores y otros sujetos aprendices, sin olvidar las salvedades indicadas respecto a la frecuencia y obligatoriedad de las interacciones personales ya comentadas.

El proceso de aprendizaje dinámico es motivador por sí mismo cuando incluye elementos metacognitivos que lo hacen consciente y supone una implicación activa del sujeto aprendiz, que puede poner a prueba sus capacidades personales de organización y personalización del aprendizaje. No se trata de la simple búsqueda de la información por caminos tecnificados, el simple "navegar" por la Web, como ya indicábamos, sino de desarrollar una actividad estructurada por vías diversas y donde se exija respuesta activa.

El conocimiento de los resultados intermedios y finales, incluyendo en este conocimiento las informaciones pertinentes para subsanar errores, constituye una faceta más del proceso didáctico, pero con mayor énfasis si cabe en el aprendizaje en solitario para que siga estando en manos del propio sujeto el control del proceso. El conocimiento de los resultados actúa así como reforzador, en terminología conductista, sin necesidad de recurrir a reforzadores específicos como los empleados en ciertos programas estrictamente lineales y que suelen adoptar forma de estímulos un tanto infantiles ${ }^{8}$.

Los elementos de motivación que de manera específica se incluyan en los programas han de tener muy presentes las características de los destinatarios, aun a sabiendas de que ello puede ser difícil cuando se pretende que los programas vayan dirigidos a públicos muy diversos. Entre estos elementos está el mismo lenguaje utilizado, que ha de procurar evitar demasiadas "familiaridades" y no dar la impresión de tratar a los estudiantes como niños de corta edad. Si hay que pecar en algún sentido más vale que sea en la dirección del respeto. Lo cual no es contradictorio con el empleo de la personalización y el lenguaje directo en muchas ocasiones, para mostrar cómo se tenía presente al destinatario en el momento de la confección de los materiales didácticos, en la línea de los textos dialógicos a que hacía referencia Holmberg (1985), siempre pensado en la parte estructurada y diseñada explícitamente para la educación a distancia, que no tienen porqué ser la totalidad de los materiales didácticos que constituyen un programa, ciertamente.

Los autores insisten en considerar que la mejor motivación es la que provoca el aprendizaje mismo, la que surge de la satisfacción de aprender. A este tipo de motivación hay que tender, sin duda, pero cuando se pretende hacer llegar los beneficios de un programa no presencial a un público amplio, donde habrá diferentes niveles de capacidad y de compromiso inicial, se hace necesario contemplar

8. Tal es el caso de aparecer muñequitos, incluir aplausos o sonidos estridentes, etc. 
incentivos intrínsecos y extrínsecos que atiendan a la diversidad de situaciones que se plantean.

Naturalmente que la naturaleza de los medios puede añadir un factor complementario de motivación. Los medios serán por sí mismos motivadores si son atractivos, ágiles y su dominio aporta un "valor añadido" al estricto aprendizaje de los. Tal es el caso del uso de las NTIC. Pero esta motivación no será suficiente por sí misma y su duración puede ser muy efímera, la del tiempo que comporta la familiarización con tales medios. Téngase presente que va en progresivo aumento el número de personas que ya manejan las NTic de manera habitual, en su domicilio o en el lugar de trabajo, por lo tanto el factor novedad puede ser nulo. En el caso de ser empleadas habitualmente en la actividad laboral pueden ser incluso un factor desmotivador, al tener los sujetos la sensación de que estudiar por estos medios equivale a prolongar su jornada laboral, como ha ocurrido en algunas empresas que las han empleado en sus programas de formación continua. Confiar la motivación en la educación a distancia exclusivamente al empleo de las NTIC sería, por tanto, un grave error.

\section{UNOS COMENTARIOS FINALES}

Con la introducción de las NTIC ya nada será como antes en la comunicación social y en la educación a distancia, porque los parámetros de tiempo y espacio diferidos en que se basa esta modalidad de educación han quedado fuertemente alterados. Pero una cosa son los cambios en los medios y otra distinta son los cambios en los paradigmas didácticos. La tecnología los posibilita pero no los comporta obligatoriamente.

La presencia de las nuevas tecnologías en la educación a distancia pone nuevamente de actualidad el debate sobre los modelos pedagógicos "cerrados" o "abiertos" que deben inspirar su uso. ¿Conviene pensar en modelos que imponen las informaciones y que se mueven bajo esquemas de autosuficiencia, o bien hay que optar decididamente por programas que son simples guías o sugerencias que el sujeto puede desestimar para buscar y organizar la información como desee?

Para responder a este dilema hay que distinguir muy claramente lo que significa un simple acceso a una información disponible, tal es el caso de Internet en general, de lo que implica un aprendizaje estructurado, donde se piden a los sujetos respuestas complejas, reflexivas y contextualizadas. Éstas no se lograrán sin un dominio de los conocimientos instrumentales básicos en cada ámbito del saber, lo cual demanda una propuesta didáctica estructurada, que sirva de modelo de las respuestas también estructuradas que se esperan de los sujetos que aprenden. Nuestra mente no actúa de forma lineal, como proponían los iniciadores de la enseñanza programada, pero el dominio de conocimientos y competencias básicas demanda de secuenciación clara y de práctica repetitiva. A partir de ahí se podrán lograr los niveles superiores dèl aprendizaje. 
Pero tampoco se puede perder de vista que los aprendizajes de nivel superior nos remiten a una concepción compleja de la realidad. Es más, se puede afirmar con rotundidad que hay que preparar a los sujetos para la comprensión de la realidad como algo complejo, de límites difusos, donde no son aplicables los esquemas cerrados de tipo algorítmico (Morín, 2000). La metodología pedagógica que hay que fomentar, sea en la educación a distancia o presencial, es aquella que ayude a los sujetos a matizar, a perseguir el rigor, bajo la convicción de que el simplismo no tiene cabida en la interpretación de la realidad, sea física o social. Sin duda hay que preparar para buscar y encontrar la información, pero también para posicionarse ante ella de manera crítica.

Por consiguiente, en un programa de educación a distancia serán precisas ambas perspectivas del aprendizaje: la que garantiza los conocimientos básicos instrumentales, bajo un modelo didáctico más estructurado - sin que por ello sea estrictamente simplista y lineal—, y los aprendizajes complejos, que se facilitan mediante esquemas abiertos, donde el protagonismo del sujeto que aprende adquiere toda plenitud.

Aún una última consideración. El contexto general de consumo en que estamos inmersos y la preponderancia absoluta de los medios de comunicación de masa en nuestra vida cotidiana llevan a que las formas bajo las que tales medios se rigen, básicamente las propias de la publicidad, la simplicidad, la forma por encima de todo, influyan en los materiales didácticos de carácter multimedia, con las consiguientes consecuencias sobre el tipo de aprendizaje logrado, que a su vez resulta también simplista y esquemático. En este contexto sintáctico de comunicación, ¿cómo lograr que el mensaje educativo provoque la necesaria reflexión personal y logre un aprendizaje estructurado y firme? Pues poniendo el énfasis, como hemos reiterado, en la estructura organizada del mensaje y en su naturaleza activa y también ética. Todo ello sin perder nunca de vista los principios básicos de racionalidad y optimización de los recursos, que también deben regir el uso de las tecnologías en el campo educativo. 


\section{BiBLIOGRAFÍA}

Álvarez, L. y otros (2000): "Del hipertexto como guía de navegación al hipertexto como procesador de información". Aula abierta, 75, 27-38.

HOLMBERG, B. (1985): Educación a distancia: situación y perspectivas. Kapelusz, Buenos Aires.

Landow, G. P. (comp.) (1997): Teoría del bipertexto. Paidós, Barcelona.

Lave, J. y Wenger, E. (1991): Situed Learning. Cambridge University Press, Cambridge.

Morin, E. (2000): La mente bien ordenada. Seix Barral, Barcelona.

PAGÉs, A. (1999): "Professionalització en entorns virtuals integrats". En DuART, J. M. y SANGrÀ, A. (eds.): Aprenentatge i virtualitat. Edicions de la uoc, Barcelona, pp. 78-89.

Rodríguez Neira, T. (2000): "Textos e hipertextos". Aula abierta, 75, 3-26.

Romiszowski, A. J. (1999): "Ensenyament i aprenentatge a distància per mitjà del Web: intent revolucionari o reacció davant la necessitat? En DuART, J. M. y SANGRÀ, A. (eds.): Aprenentatge i virtualitat. Edicions de la UOC, Barcelona, pp. 17-34.

Sarramona, J. (1999): "La autoformación en una sociedad cognitiva". Revista Iberoamericana de Educación a Distancia, 2 (1), junio, 41-59.

VYGotSKY, L. S. (1973): "Aprendizaje y desarrollo intelectual en la edad escolar". Psicologia y Pedagogía. Akal, Madrid, pp. 23-39.

WHEELER, S. (2000): "The traditional university is dead: long live the distributed university". European Universities Continuing Education Conference. University of Bergen (Norway). 